\title{
A Wavelength- and Loss-Tunable Band-Rejection Filter Based on Corrugated Long-Period Fiber Grating
}

\author{
C. Y. Lin and Lon A. Wang
}

\begin{abstract}
We demonstrate a long-period fiber grating composed of an etched corrugated structure that can be used as a wavelength- and loss-tunable band-rejection filter. The tunabilities are based on the index modulation capable of being varied in the corrugated structure under externally applied mechanical forces. The new type of fiber filter enables wavelength and loss tuning ranges of more than $40 \mathrm{~nm}$ and $25 \mathrm{~dB}$ by adjusting the applied amounts of torsion and tensile forces, respectively.
\end{abstract}

Index Terms-Corrugated structure, fiber gratings, tunable filters.

\section{INTRODUCTION}

$\mathbf{R}$ ECENTLY, long-period fiber gratings (LPFGs) have attracted much attention because they can be used as gain equalizers for broad-band amplifiers and as band-rejection filters [1], [2]. Furthermore, an active control on their transmission spectra made it possible to maintain the gain flatness or the equal output signal level in an optical fiber network even when an input signal varies, thus making LPFG-based tunable filters of great interest. It is noted that the fabrication method for most conventional LPFGs is based on the photosensitivity of fiber core to ultraviolet light. Both wavelengths and losses at the resonance peaks of LPFGs are therefore determined by the fabrication process and can hardly be tuned, which may limit the applications of LPFGs. Recently considerable efforts have been made to expand the tunabilities of an LPFG. It was reported that the LPFG could have a large wavelength tuning range using enhanced temperature sensitivity [3]; however, its peak loss could not be tuned. Similarly, the resonance loss of the LPFG based on quadratic-dispersion gratings could be varied by strain [4], but not the resonance wavelength. Although an all-fiber acoustooptic filter was shown to have wavelength and loss tunabilities, the need of a silicon horn and the associated control mechanisms may be complicated [5].

In this letter, we describe a new type of LPFG made of a corrugated structure over which periodical strain distributions are induced when external torsion or tensile force is applied. We have shown that such a corrugated LPFG can be served as a

Manuscript received May 23, 2000; revised December 27, 2000.

C. Y. Lin is with the Department of Electrical Engineering and Institute of Electro-Optical Engineering, National Taiwan University, Taipei, Taiwan, R.O.C.

L. A. Wang is with the Department of Electrical Engineering and Institute of Electro-Optical Engineering, National Taiwan University, Taipei, Taiwan, R.O.C. and also with the Department of Electrical Engineering, National Taiwan University, Taipei, Taiwan, R.O.C. (e-mail: lon@ccms.ntu.edu.tw).

Publisher Item Identifier S 1041-1135(01)03130-5.

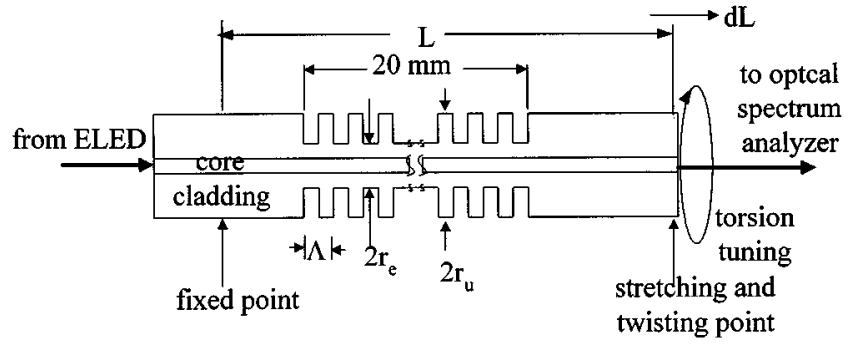

Fig. 1. Schematic diagram showing our LPFG made of the corrugated structure and its measurement setup.

loss-tunable band-rejection filter by adjusting the amount of the applied tensile strain [6]. Here, we experimentally demonstrate that, for the first time to the best of our knowledge, this type of band-rejection filter is capable of tuning the resonant wavelength and the loss by adjusting the amounts of applied torsion and tensile forces.

\section{PRINCIPLE OF OPERATION}

The corrugated LPFG is schematically shown in Fig. 1. It consists of two unit sections. One is the etched region with cladding radius $r_{\mathbf{e}}$, and the other is the unetched region with radius $r_{\mathbf{u}}$. When a tensile force is applied along the fiber in an equilibrium condition, each of the two regions will experience different tensile strain that is inversely proportional to the cross section area of the corresponding region. Therefore, by photoelastic effect, the differential strain results in periodic index modulation. Since the coupling coefficient $\kappa$ between the core and cladding modes increases with the index modulation and the transmission of the LPFG can be expressed as [2]

$$
T=\cos ^{2}(\kappa l)
$$

where $l$ is the length of the corrugated LPFG, then, the resonant loss can be tuned by the applied tensile force. The structure, thus, can act as a tunable loss filter.

It has been shown that the peak resonant wavelength $\lambda$ at which the resonant coupling occurs can be written as [6]:

$$
\lambda \approx \lambda_{\text {res }}\left(1+\frac{\left(\delta n_{\text {core }}-\delta n_{\text {clad }}\right)\left(d \lambda_{\text {res }} / d \Lambda\right)}{\left(n_{\text {core }}-n_{\text {clad }}\right)^{2}}\right)
$$

where $\lambda_{\text {res }}$ is the initial resonant wavelength; $n_{\text {core }}$ and $n_{\text {clad }}$ are effective indices of fundamental core and cladding modes, respectively; $\Lambda$ is the grating period; and $\delta n_{\text {core }}$ and $\delta n_{\text {clad }}$ are the index changes in the core or cladding regions, respectively. 
For the corrugated LPFG, since the Young's modulus is nearly uniform in the cross section of a fiber, the resultant tensile strain is not a strong function of the cross section. As a result, the index changes induced in both core and cladding over the entire cross section in our LPFG are similar. From (2), the difference $\left(\delta n_{\text {core }}-\delta n_{\text {clad }}\right)$ as induced in a corrugated LPFG is then much smaller than $\delta n_{\text {core }}$ as induced in a conventional LPFG, which may result in much less wavelength shifting in the corrugated LPFG [7].

Next, we consider the effect caused by twisting the corrugated LPFG. For a uniform rod subject to an applied torque, the twist rate (i.e., torsional angle per length) is inversely proportional to the fourth power of the radius [8]. For the corrugated structure, the twist rates are different in the etched and unetched regions due to the difference of the corresponding cladding radii, which involves the analysis of the strain field distribution under torsion. Detailed results will be reported in the future publication. Apart from that induced by the tensile strain, another induced periodical index modulation is expected. Significant excess stresses around the discontinuity areas exist. Since the corresponding strain fields are mainly distributed within the cladding rather than the core regions, the contribution to the index change $\delta n_{\text {clad }}$ is much larger. From (2), the peak resonant wavelength shifts with the increasing twist rate, which forms the basis of wavelength tunability. Some co-related strain distribution caused by twist have the same periodic variation and will induce additional index modulation; therefore, it may contribute to coupling among core and cladding modes and result in the change of the resonant transmission loss. Owing to the similarity of $\delta n_{\text {core }}$ and $\delta n_{\text {clad }}$ induced by the applied tensile strain [6], it is expected that the resonance wavelength is almost dominated by torsion even when combined with a tensile strain. Thus, a loss-tunable band-rejection filter whose resonance wavelength can be tuned by twisting is formed.

\section{EXPERIMENTAL RESULTS}

A dispersion-shifted fiber (DSF) with an original cladding radius of $62.5 \mu \mathrm{m}$ is used. The corrugated structure is built by etching a pre-patterned fiber using hydrofluoric acid solution [6]. It has a length of $20 \mathrm{~mm}$ with an etched radius of $\sim 20 \mu \mathrm{m}$ and a period of $\sim 400 \mu \mathrm{m}$. The experiment setup for characterizing wavelength and loss tunabilities of the LPFG is also depicted in Fig. 1. A tensile force and an external torsion torque are applied to inducing an average tensile strain $d L / L$ and an average twisting rate $\tau=2 \pi N / L(\mathrm{rad} / \mathrm{m})$, respectively. $d L$ and $N$ are the total elongation and the number of torsional turns over the distance $L$ between the fixed and the stretching points. To facilitate a precise control on the adjustment of $\tau$ and $d L / L, L$ is set as long as $1 \mathrm{~m}$ owing to the precision limitation of our mechanical stages.

The evolution of transmission spectra of the LPFG at three different fixed twist rates for increasing tensile forces is shown in Fig. 2. Three noteworthy characteristics are observed. First, all transmission losses at the resonance peaks mainly increase with induced tensile strain, which indicates $\kappa$ increases with tensile strain as described in (1). Second, for each applied twist rate, the shift of their resonance wavelengths is very small, less

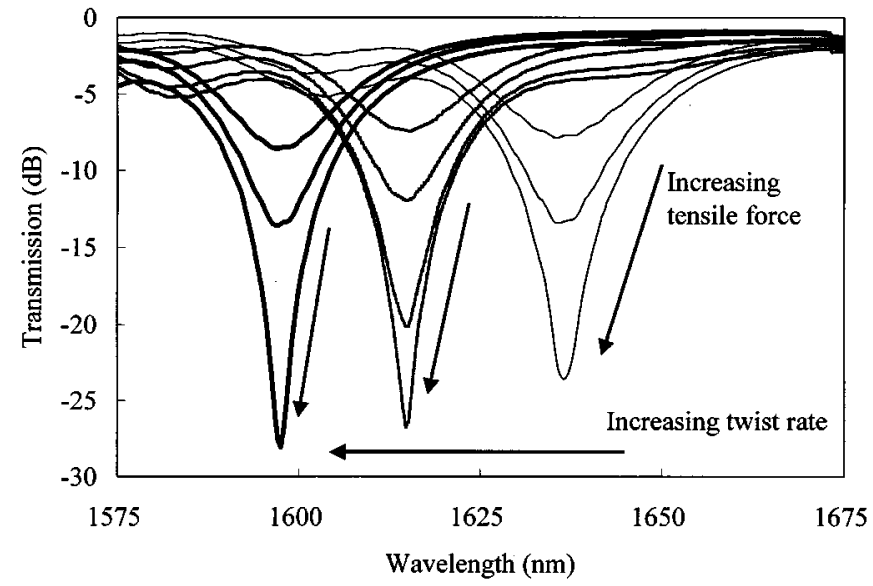

Fig. 2. Evolution of transmission spectra of the corrugated LPFG when different twist rates combined with increasing tensile strains are applied.

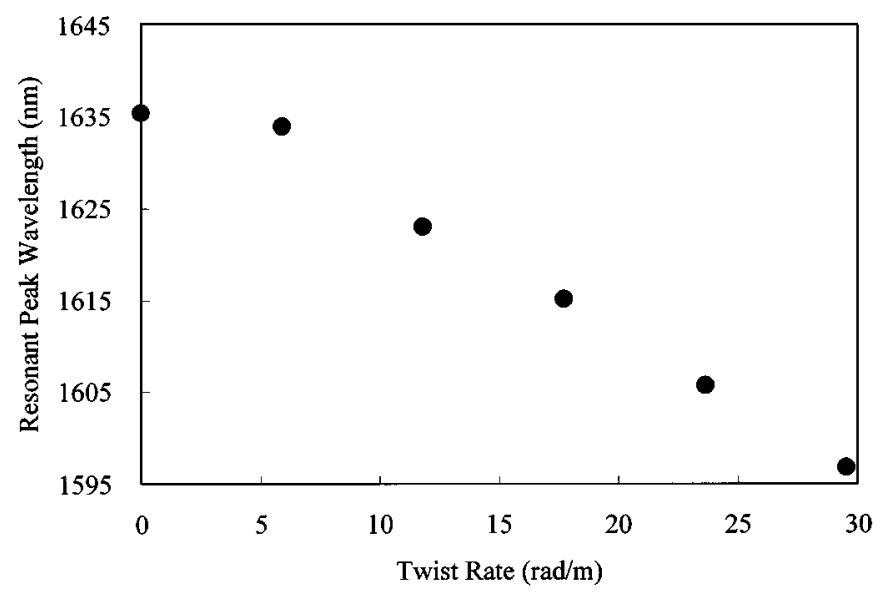

Fig. 3. Variation of resonant peak wavelength with the applied twist rate.

than $2 \mathrm{~nm}$ for each entire loss tuning range. This characteristic is unique as compared to the conventional LPFGs made by photoexposure that exhibit a considerable amount of wavelength shift during the growth of index modulation [7]. As indicated in (2), such a characteristic is attributed to the similarity between $\delta n_{\text {core }}$ and $\delta n_{\text {clad }}$ caused by tensile strain. Third, the resonance wavelength shift is determined by the twist rate only, and moves toward the shorter wavelength side with increasing twist rate. These phenomena are all consistent with those described in Section II.

The variation of resonance wavelength with applied twist rate is shown in Fig. 3. The wavelength can be tuned $\sim 40 \mathrm{~nm}$ at a twist rate of $\sim 30 \mathrm{rad} / \mathrm{m}$. We note that for a conventional LPFG resulted from photosensitivity, no obvious change in transmission spectra is measured even when the twist rate is doubled. Therefore, such a special characteristic is attributed to the corrugated structure of the LPFG. A rigorous analysis in this regard is currently being pursued for theoretical verification.

Loss tuning at different twist rates is shown in Fig. 4, and the trend is consistent with that described in (1). When no twist rate or strain is applied, the initial insertion loss is less than $0.5 \mathrm{~dB}$ indicating a negligible waveguide effect resulted from the corrugated structure. As the tensile strains increase, the maximum losses can exceed $30 \mathrm{~dB}$ when $d L / L$ is less than $600 \mu \varepsilon$. Note 


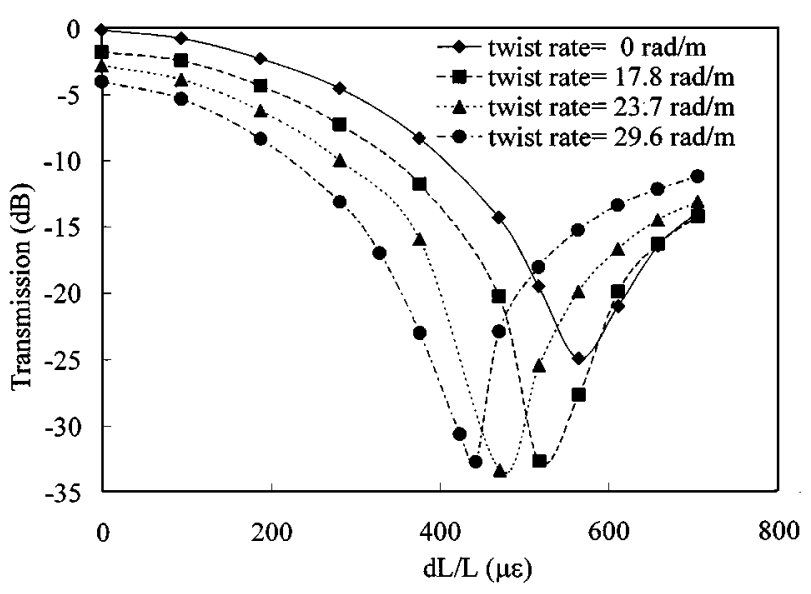

Fig. 4. Peak transmission losses versus the applied strains when combined with different twist rates.

that as further strain is applied, the transmission loss decreases due to the reverse coupling from the cladding mode to the core mode, which is similar to the over-exposure case as commonly observed in the fabrication of photo-induced LPFGs. It is also noted that the more twist rate is applied the less tensile strain is needed to reach the maximum loss owing to additional coupling strength induced by torsion. Additionally, no measurable degradation is detected after more than 50-cycle measurements on the same corrugated LPFG.

\section{CONCLUSION}

We demonstrate that the tunabilities of wavelength and loss of a corrugated LPFG are obtained by adjusting the applied tor- sion and tensile forces, respectively, using a simple mechanical setup. Since the shift of resonant peak wavelength is very small $(<2 \mathrm{~nm})$ during the overall loss tuning, a specified resonant peak wavelength and loss can be obtained easily by first adjusting the amounts of torsion and then tensile forces. More than $40 \mathrm{~nm}$ and $25 \mathrm{~dB}$ can be tuned without residual stress. Such a tunable band-rejection filter is expected to be useful in various applications in fiber communication.

\section{REFERENCES}

[1] Y. Sun, J. W. Sulhoff, A. K. Srivastava, A. A. Abramov, T. A. Strasser, P. F. Wysocki, J. R. Pedrazzani, J. B. Judkins, R. P. Espindola, C. Wolf, J. L. Zyskind, A. M. Vengsarkar, and J. Zhou, "A gain-flattened ultra wide band EDFA for high capacity WDM optical communications systems," in Proc. ECOC'98, Madrid, Spain, Sept. 1998, pp. 53-54.

[2] A. M. Vengsarkar, P. J. Lemaire, J. B. Judkins, V. Bhatia, T. Erdogan, and J. E. Spie, "Long-period fiber gratings as band-rejection filters," $J$. Lightwave Technol., vol. 14, pp. 58-65, Jan. 1996.

[3] A. A. Abramov, B. J. Eggleton, J. A. Rogers, R. P. Espindola, A. Hale, R. S. Windler, and T. A. Strasser, "Electrically Tunable Efficient Broad-Band Fiber Filter," Photon. Technol. Lett., vol. 11, pp. 445-447, Apr. 1999.

[4] V. Grubsky and J. Feinberg, "Loss-period fiber gratings with variable coupling for real-time sensing applications," Opt. Lett., vol. 25, no. 4, pp. 203-205, 2000.

[5] R. Feced, C. Alegria, M. N. Zervas, and R. I. Laming, "Acoustooptic attenuation filters based on tapered optical fibers," J. Select. Topics Quantum Electron., vol. 5, pp. 1278-1288, Sept./Oct. 1999.

[6] C. Y. Lin and L. A. Wang, "Loss-tunable long period fiber grating made from etched corrugation structure," Electron. Lett., vol. 35, no. 21, pp. 1872-1873, 1999.

[7] T. W. MacDougall, S. Pilevar, C. W. Haggans, and M. A. Jackson, "Generalized expression for the growth of long period gratings," IEEE Photon. Technol. Lett., vol. 10, pp. 1449-1451, Oct. 1998.

[8] Beer and Johnston, Mechanics of Materials. Toronto, Canada: McGraw-Hill Ryerson, 1985. 